http://jmscr.igmpublication.org/home/ ISSN (e)-2347-176x ISSN (p) 2455-0450 crossref DOI: https://dx.doi.org/10.18535/jmscr/v9i4.03

\title{
Albumin- Bilirubin (ALBI) Score a New and Simple Bedside Model to Predict Mortality in Patients with Cirrhosis
}

\author{
Authors \\ Ravikumar. P ${ }^{1}$, Caroline Selvi ${ }^{2}$, Premkumar ${ }^{3}$, A. Chezhian ${ }^{4}$, R Murali $^{5}$, I. Shubha ${ }^{6}$ \\ Madras Medical College, Chennai, India
}

\begin{abstract}
Aim: The existence of reliable prognostic indices is of paramount importance in the management of cirrhosis. The albumin-bilirubin (ALBI) score is a new model for assessing the severity of liver dysfunction. The aim of this study was to determine the ALBI score's mortality prediction among cirrhotics and its comparison with MELD, CTP scores.

Methods: This prospective study included one hundred cirrhotics where we calculated CTP, MELD and ALBI scores were calculated within 24 hours of admission. The mortality was assessed during the hospital stay and follow-up at 2, 4 and 6 months. The diagnostic accuracy of CTP, MELD and ALBI scores for mortality in cirrhotic patients was determined by receiver operating characteristic curve (ROC) analysis.

Results: In the overall analysis, all of the three scores were significantly assess the mortality (CTP $A U C=0.917$, 95\% CI: 0.857-0.977, P<0.0001; MELD AUC=0.935, 95\% CI: 0.887-0.982, P<0.0001; ALBI AUC $=0.964,95 \%$ CI: 0.924-1.003, P<0.0001). The best cut-off values for CTP, MELD and ALBI were at $>12.5$ (sensitivity $=75.86 \%$, specificity $=97.18 \%),>22.5($ sensitivity $=100 \%$, specificity $=$ $84.51 \%)$ and $>-1.38$ (sensitivity $=100 \%$, specificity $=94.37 \%)$ with diagnostic accuracy of $91 \%, 89 \%$ and $96 \%$ respectively.

Conclusion: The fact that ALBI score uses only two convenient parameters, readily obtained by easily accessible blood tests, objectively evaluated and being non inferior to CTP and MELD scores indicates that ALBI score might be a better mortality predictor in cirrhosis. Therefore, all these favour its applicability in clinical practice as a substitute for the CTP and MELD scores.

Keywords: Bilirubin, Albumins, Prognosis, Liver Cirrhosis.
\end{abstract}

\section{Introduction}

Liver cirrhosis is a common cause of death in the world $^{[1,2]}$. The accurate prognostication of liver cirrhosis is important in our daily practice. The existence of reliable prognostic indices is important in the management of cirrhosis ${ }^{[3,4,5]}$. Child-TurcottePugh (CTP) score and Model for End-Stage Liver Disease (MELD) scores are available for assessing liver function and the severity of liver injury. The most commonly used tool to predict the prognosis of liver cirrhosis is Child-Pugh score ${ }^{[4]}$. However, it has been established for a long time, and its components are selected primarily based on the surgeons' experiences. Model for end-stage liver disease (MELD) score is another tool for prognostic assessment of liver cirrhosis ${ }^{[5,6]}$. Until now, there are lots of controversy regarding the comparison of Child-Pugh versus MELD scores ${ }^{[7-10]}$. The albumin- 
bilirubin (ALBI) score is a new model for assessing the severity of liver dysfunction.

It is a novel, simple, and readily available model calculated using mathematical formula:

$0.85(\mathrm{alb} g / \mathrm{l})+0.66 \mathrm{X} \log (\mathrm{bil}$ umol/l) .

Compared to CTP score, there are several advantages of the ALBI score. First, the two parameters, serum albumin and serum bilirubin for the ALBI score, are obtained by accessible blood tests. But the clinical assessment of ascites and hepatic encephalopathy for the CTP score is difficult to score consistently. Moreover, different grades of ascites are not uniform in CTP score ${ }^{[4,11]}$. Second, the ALBI score is selected on the basis of a mathematical model but the five components of the CTP score are selected empirically ${ }^{[12]}$. Third, the values of bilirubin in the CTP score should be modified for chronic cholestatic diseases such as primary biliary cirrhosis for better result ${ }^{[13]}$.

For cirrhotic patient the sensitivity and specificity of CTP score is $90 \%$ and $33 \%$ but for ALBI score is $84 \%$ and $39 \%^{[8]}$. Another study support that sensitivity and specificity of ALBI score in liver cirrhosis patient with upper gastrointestinal bleeding is $92.86 \%$ and $64.01 \%$ respectively but in case of CTP score sensitivity and specificity is $57.14 \%$ and $86.24 \%$ respectively ${ }^{[9,15]}$.

Considering the above scenario, the newly developed ALBI score is more convenient than CTP score. ALBI score are simple, repeatable, less expensive, can be done easily even in peripheral laboratory, without the need for special tests.

This new score may be a better tool than CTP score in cirrhotic patients for early management and will be helpful for quick decision making for clinician in near future and beneficial for cirrhotic patients.

\section{Aim}

The aim of this study was to determine the ALBI score's mortality prediction among cirrhotics and its comparison with MELD and CTP scores.

\section{Methodology}

This prospective study of one hundred cirrhotics who were admitted in RGGGH, chennai from July
2019 to Jan 2020. Age, sex, cause of cirrhosis, compensation status, age at diagnosis of cirrhosis, first complications of decompensated cirrhosis, existence or appearance of hepatocellular cancer, as well as biochemical variables were recorded for all patients. We calculated CTP, MELD and ALBI scores were calculated within 24 hours of admission. The mortality was assessed during the hospital stay and follow-up at 2, 4 and 6 months.

All statistical analysis was performed using MEDCALC software. Continuous data were expressed as the mean $+/-\mathrm{SD}$ and median with minimum and maximum. ROC curve were performed to identify the discriminative ability of ALBI, MELD and CTP score in predicting mortality. AUC were calculated and compared. The best cut off value was selected as the sum of sensitivity and specificity was maximal. The sensitivity, specificity, positive likelihood ratio, negative likelihood ratio were reported.

\section{Results}

In 100 cirrhotic patients, age distribution was between 26-87 years with mean age of patient being $47.85 \pm 14.34$ years. Sixty four were males and 36 were females. Among 100 patients, 29 patients had mortality over period 6 months follow-up.

Table 1: Etiology of Cirrhosis

\begin{tabular}{|l|c|c|}
\hline ETIOLOGY & NUMBER & PERCENTAGE \\
\hline ALCOHOL & 42 & 42.0 \\
\hline CRYPTOGENIC & 10 & 10.0 \\
\hline NAFLD & 14 & 14.0 \\
\hline VIRAL + ALCOHOL & 10 & 10.0 \\
\hline VIRAL & 24 & 24.0 \\
\hline TOTAL & 100 & 100.0 \\
\hline
\end{tabular}

Table 2: Mortality during the follow-up of $2^{\text {nd }}, 4^{\text {th }}$ and $6^{\text {th }}$ Month

\begin{tabular}{|l|c|c|}
\hline MORTALITY & NUMBER & PERCENTAGE \\
\hline SURVIVED & 71 & 71.0 \\
\hline DIED AT 2ND MONTH & 8 & 8.0 \\
\hline DIED AT 4TH MONTH & 8 & 8.0 \\
\hline DIED AT 6TH MONTH & 13 & 13.0 \\
\hline TOTAL & 100 & 100.0 \\
\hline
\end{tabular}


Table 3: ROC Analysis to Predict Overall Mortality

\begin{tabular}{|l|c|c|c|c|}
\hline \multicolumn{5}{|l|}{ AREA UNDER THE CURVE } \\
\hline $\begin{array}{l}\text { VARIA } \\
\text { BLE }\end{array}$ & AREA & $\begin{array}{c}\text { STD. } \\
\text { ERROR }\end{array}$ & $\begin{array}{c}\text { P } \\
\text { VALUE }\end{array}$ & $\begin{array}{c}95 \% \\
\text { CONFIDENCE } \\
\text { INTERVAL }\end{array}$ \\
\hline MELD & .935 & .024 & $<0.001$ & $.887-.982$ \\
\hline CTP & .917 & .031 & $<0.001$ & $.857-.977$ \\
\hline ALBI & .964 & .020 & $<0.001$ & $.924-1.003$ \\
\hline
\end{tabular}

Table 4: Comparison of Best Cut-Off values for MELD, CTP AND ALBI Scores along with their Sensitivity, Specificity and Diagnostic accuracy in Predicting Mortality of Cirrhotic Patients

\begin{tabular}{|l|c|c|c|c|}
\hline $\begin{array}{l}\text { VARIA } \\
\text { BLE }\end{array}$ & $\begin{array}{c}\text { CUT } \\
\text { OFF }\end{array}$ & $\begin{array}{c}\text { SENSITI } \\
\text { VITY }\end{array}$ & $\begin{array}{c}\text { SPECIFI } \\
\text { CITY }\end{array}$ & ACCURACY \\
\hline MELD & $\geq 22.5$ & $100 \%$ & $84.51 \%$ & $89 \%$ \\
\hline CTP & $\geq 12.5$ & $75.86 \%$ & $97.18 \%$ & $91 \%$ \\
\hline ALBI & $\geq-1.385$ & $100 \%$ & $94.37 \%$ & $96 \%$ \\
\hline
\end{tabular}

Table 5: AUC for Predicting the Mortality Was Not Significantly Different Between the Childs PUGH, MELD and ALBI Scores

\begin{tabular}{|c|c|}
\hline \multicolumn{2}{|l|}{ ALBI $\sim$ CTP } \\
\hline Difference between areas & 0.0471 \\
\hline Standard Error & 0.0305 \\
\hline 95\% Confidence Interval & -0.0127 to 0.107 \\
\hline z statistic & 1.544 \\
\hline Significance level & $\mathbf{P}=\mathbf{0 . 1 2 2 7}$ \\
\hline \multicolumn{2}{|l|}{ ALBI $\sim$ MELD } \\
\hline Difference between areas & 0.0291 \\
\hline Standard Error & 0.0150 \\
\hline 95\% Confidence Interval & -0.000246 to 0.0585 \\
\hline z statistic & 1.944 \\
\hline Significance level & $P=0.0519$ \\
\hline \multicolumn{2}{|l|}{ CTP MELD } \\
\hline Difference between areas & 0.0180 \\
\hline Standard Error & 0.0278 \\
\hline $95 \%$ Confidence Interval & -0.0365 to 0.0724 \\
\hline z statistic & 0.647 \\
\hline Significance level & $P=0.5179$ \\
\hline
\end{tabular}

Figure 1: Showing Comparison of ROC Curves

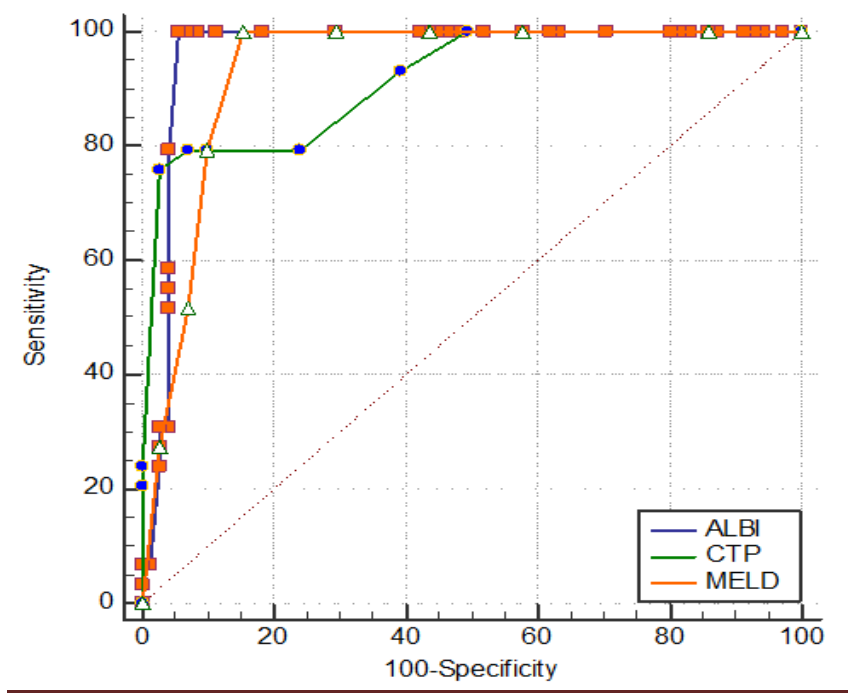

All scoring systems were found to have diagnostic accuracy in predicting survival $(\mathrm{P}<0.001)$. ALBI had the optimum balance between sensitivity and specificity (AUC 0.964, 95\%CI 0.924-1.003) compared with the other scores.

\section{Discussion}

Child pugh score and MELD score have been studied extensively for their prognostic abilities and have shown to perform good to predict the mortality of cirrhotic patients. But the cumbersome calculation of scores and the variables included in them have subjective variability has led to the development of ALBI score.

ALBI score involves only two variables and already studied in various liver disorders such as HBV, HCC, and Primary Biliary Cirrhosis and has shown to perform well and is comparable with the Child pugh and MELD scores.

In our study attempt has been made to compare the discriminative ability of ALBI score with that of the Child pugh score and MELD score in predicting the mortality in cirrhosis patients. ALBI score showed better performance compared to Child pugh score and MELD score in predicting mortality but there was no statistical difference between them.

In a study by Lichun Shao et al, ALBI score had similar prognostic ability in predicting mortality in cirrhotic patients as that of Child pugh and MELD score. It also suggests that ALBI score can be readily used as prognostic model ${ }^{[16]}$.

A study by Bo Chen et al evaluated CP score, MELD, and ALBI in predicting 3-month mortality in patients with acute-on-chronic liver failure and reported that both ALBI and MELD scores were independent predictors $(\mathrm{P}<0.001)$. However, the ROC curves indicated that the MELD score was better than the ALBI score (AUC 0.837 and 0.784, respectively) ${ }^{[17]}$.

A study by Zou et al [15] evaluated the in-hospital mortality in relation to ALBI, CTP and MELD scores in 631 cirrhotic patients and found that the ALBI score had the best AUC (0.808, 0.785, and 0.787 respectively). This study showed that ALBI is a good indicator of short-term prognosis, in 
agreement with our study and best assessed by ALBI (AUC 0.964).

Peng et al study showed that there was no significant difference in predicting in-hospital mortality in cirrhotic patients. ${ }^{[14]}$

A retrospective Study done by Xavier SA et al, on 111 patients concluded that ALBI score is particularly useful in the assessment of short come outcomes, with a better performance than the most commonly used scores ${ }^{[18]}$.

Hsieh YC et al conducted study in Taiwan included 242 patients with both compensated and decompensated cirrhosis concluded that both ALBI and MELD scores significantly predicted 3-month and 6-month mortality (AUC $0.773,0.691$ vs. 0.813, 0.740 , respectively ${ }^{[19]}$.

Our study has the limitation that it was conducted in a single tertiary institute with a relative small number of cases. Therefore, a larger cohort of well stratified cirrhotics of different etiologies should be studied in order to confirm our findings. Moreover, there was an imbalance in the compensation status of our patients, as more than $70 \%$ already had decompensated cirrhosis when they were enrolled in our study.

Nonetheless, our study has demonstrated that ALBI is an overall accurate and reliable score in decompensated cirrhosis, irrespective of etiology, equal to or better than the other predictive scores in predicting mortality. Therefore, in view of its simplicity, it can confidently replace the other more complex scores.

\section{Conclusion}

The fact that ALBI score uses only two convenient parameters, readily obtained by easily accessible blood tests, objectively evaluated and being non inferior to CTP and MELD scores indicates that ALBI score might be a better mortality predictor in cirrhosis.

Therefore, all these favour its applicability in clinical practice as a substitute for the CTP and MELD scores.

\section{References}

1. Blachier M, Leleu H, Peck-Radosavljevic M, Valla D-C and Roudot-Thoraval F. The burden of liver disease in Europe: A review of available epidemiological data. Journal of Hepatology 2013; 58(3):593-608.

2. Lozano R, Naghavi M, Foreman K, Lim S, Shibuya K, Aboyans V, et al. Global and regional mortality from 235 causes of death for 20 age groups in 1990 and 2010: a systematic analysis for the Global Burden of Disease Study 2010. Lancet 2012; 380:20952128.

3. Child CG, Turcotte JG. Surgery and portal hypertension. Major Probl Clin Surg 1964;1:1-85.

4. Pugh RN, Murray-Lyon IM, Dawson JL, Pietroni MC, Williams R. Transection of the oesophagus for bleeding oesophageal varices. Br J Surg 1973;60:646-649.

5. Kamath $\mathrm{P}$, Wiesner $\mathrm{R}$, Malinchoc $\mathrm{M}$, Kremers W, Therneau T, Damico G, et al. A model to predict survival in patients with end-stage liver disease. Gastroenterology 2001; 120(5).

6. Kamath PS and Kim WR. The model for end-stage liver disease (MELD). Hepatology 2007;45(3):797-805. Child CG, Turcotte JG. Surgery and portal hypertension. In: Child CG (editor): The liver and portal hypertension. Saunders: Philadelphia; 1964, pp. 50-64.

7. Gheorghe L, Iacob S, Simionov I, Vandan $\mathrm{R}$, Gheoghe C, Iacob R, et al. Natural history of compensated viral $\mathrm{B}$ and $\mathrm{D}$ cirrhosis. Rom J Gastroenterol 2005; 14:329-335.

8. Cholongitas E, Marelli L, Shusang V, Senzolo M, Rolles K, Patch D, et al. A systematic review of the performance of the model for end-stage liver disease (MELD) in the setting of liver transplantation. Liver Transplantation 2006; 12(7):1049-1061.

9. Peng Y, Qi X, Dai J, Li H and Guo X. Child-Pugh versus MELD score for predicting the in-hospital mortality of acute 
upper gastrointestinal bleeding in liver cirrhosis. Int J Clin Exp Med 2015; 8:751757.

10. Chalasani N, Kahi C, Francois F, Pinto A, Marathe A, Bini EJ, et al. Model for endstage liver disease(MELD) for predicting mortality in patients with acute variceal bleeding. Hepatology 2002; 35(5):12821284.

11. Infante-Rivard C, Esnaola S, Villeneuve JP. Clinical and statistical validity of conventional prognostic factors in predicting short-term survival among cirrhotics. Hepatology. 1987; 7 (4): 660-5.

12. Arroyo V. Pathophysiology, diagnosis and treatment of ascites in cirrhosis. Annals of hepatology. 2002; 1 (2): 72-9.

13. Chan AW, Chan RC, Wong GL, et al. Evaluation of histological staging systems for primary biliary cirrhosis: correlation with clinical and biochemical factors and significance of pathological parameters in prognostication. Histopathology. 2014; 65: 174-86.

14. Peng Y, Qi X, Tang S, Deng H, Li J, Ning Z, Dai J, Hou F,Zhao J, Wang R, Guo X. Child-Pugh, MELD, and ALBI scores for predicting the in-hospital mortality in cirrhotic patients with acute-on-chronic liver failure. Expert review of gastroenterology \& hepatology. 2016; 10 (8): 971-80.

15. Zou D, Qi X, Zhu C, Ning Z, Hou F, Zhao J, Peng Y, Li J Deng H, Guo X. Albuminbilirubin score for predicting the in hospital mortality of acute upper gastrointestinal bleeding in liver cirrhosis: A retrospective study. Turk J Gastroenterol. 2016 Mar 1; 27 (2): 180-86.

16. Shao L, Han B, An S, Ma J, Guo X, Romeiro FG, et al. Albumin-to-bilirubin score for assessing the in- hospital death in cirrhosis. Translational Gastroenterology and Hepatology 2017;88.

17. Chen B and Lin S. Albumin-bilirubin (ALBI) score at admission predicts possible outcomes in patients with acute-on-chronic liver failure. Medicine 2017;96(24).

18. Xavier SA, Vilas-Boas R, Carvalho PB, Magalhães JT, Marinho CM and Cotter JB. Assessment of prognostic performance of Albumin-Bilirubin, Child-Pugh, and Model for End-stage Liver Disease scores in patients with liver cirrhosis complicated with acute upper gastrointestinal bleeding. European Journal of Gastroenterology \& Hepatology 2018;1.

19. Hsieh YC, Lee KC, Wang YW, et al. Correlation and prognostic accuracy between noninvasive liver fibrosis markers and portal pressure in cirrhosis: Role of ALBI score. PLoS One 2018;13:e208903. 